\title{
Minimally invasive photoacoustic imaging for device guidance and monitoring of radiofrequency ablation
}

Kalloor Joseph, Francis, Kruit, Hindrik, Rascevska, Elina, Manohar, Srirang

Francis Kalloor Joseph, Hindrik Kruit, Elina Rascevska, Srirang Manohar, "Minimally invasive photoacoustic imaging for device guidance and monitoring of radiofrequency ablation," Proc. SPIE 11240, Photons Plus Ultrasound: Imaging and Sensing 2020, 112405F (17 February 2020); doi: $10.1117 / 12.2546018$

SPIE. Event: SPIE BiOS, 2020, San Francisco, California, United States 


\title{
Minimally invasive photoacoustic imaging for device guidance and monitoring of radiofrequency ablation
}

\author{
Kalloor Joseph Francis ${ }^{\mathrm{a}}$, Hindrik Kruit ${ }^{\mathrm{a}}$, Elina Rascevska ${ }^{\mathrm{a}}$, and Srirang Manohar ${ }^{\mathrm{a}}$ \\ ${ }^{a}$ Biomedical Photonic Imaging group and Multi-Modality Medical Imaging group, Technical \\ Medical Center, University of Twente, The Netherlands
}

\begin{abstract}
Radiofrequency ablation (RFA) procedures for liver cancer treatment are hindered by high tumor recurrence. This is thought to be due to the intrinsic limitation of the heating mechanism and insufficient real-time feedback from imaging modalities. Most RFA procedures are performed under ultrasound (US) imaging and there are limitations in accurate device guidance and ablation monitoring. We propose photoacoustic (PA) imaging as a potential add-on to US imaging to address these limitations. Specifically, we present two interstitial PA imaging methods. Firstly, an annular fiber probe that can encapsulate an RFA device in its lumen. This device enables RFA device guidance, visualization of major blood vessels and targeting tumor tissue. Secondly, we used a cylindrical diffuser-based interstitial illumination to differentiate coagulated and native tissue. We present our results on RFA device guidance and ablation visualization using these approaches. The contrast provided by PA imaging for RFA needle and multiple electrodes is compared against that of US images. The difference between coagulated and native ex vivo liver tissue using PA imaging is studied. Finally, we propose a protocol to incorporate the minimally invasive PA imaging for the clinical RFA procedures. We would like to conclude with a note on how the proposed approach can potentially improve the outcome of RFA procedures.
\end{abstract}

Keywords: Photoacoustic, Ultrasound, Radiofrequency ablation, Interstitial illumination, Diffuser, Annular fiber

\section{INTRODUCTION}

Radiofrequency ablation (RFA) has gained widespread clinical acceptance for the treatment of primary and metastatic malignancies in the liver. ${ }^{1} \mathrm{RF}$ ablation involves the application of alternating current into the tumor, inducing Joule heating which leads to coagulation necrosis of tissue. ${ }^{2}$ The common clinical practice is to use computed tomography or magnetic resonance imaging for pre-operative planning and post-operative treatment assessment, and ultrasound (US) for real-time RFA needle positioning and monitoring of treatment. ${ }^{3}$ The goal of this interventional procedure is to accurately position the RFA needle at the center of the tumor, deploy the multiple electrodes into the tissue and heat the tumor to coagulation with a margin of $1 \mathrm{~cm}$ around the tumor. There are limitations to US imaging for this application. First, the visibility of the needle positioned at an angle is poor. This is mainly due to the directional nature of the transducer. Second, the treatment is monitored by observing the bubble formation during thermal treatment. ${ }^{4}$ However, shadowing from the bubbles limits visibility at the posterior region of the tumor. Additionally, large blood vessels can remove heat from the ablation zone resulting in incomplete ablation, which is also hard to monitor using US imaging. Hence there is a need for enhancing the US imaging for accurate RFA device guidance and monitoring of ablation.

An ideal modality for RFA application should be able to imaging metal needles, blood vessels and differentiate native and ablated tissue. Interestingly, the optical absorption of the metal, blood vessels, ablated and native tissue are distinct which can be utilized for optical imaging. ${ }^{5}$ In this work, we have explored the possibility of using photoacoustic (PA) imaging for RFA applications. PA imaging uses pulsed light to induce thermoelastic expansion in optical absorbers to produce acoustic waves. ${ }^{6-8}$ This modality has shown promise in imaging metal needles and is widely used to visualize vascular structures. ${ }^{9,10}$ Additionally, there is a significant difference in the

Further author information:

K. J. Francis.: E-mail: f.kalloorjoseph@utwente.nl, Telephone: +31534891559

Photons Plus Ultrasound: Imaging and Sensing 2020, edited by Alexander A. Oraevsky, Lihong V. Wang, Proc. of SPIE Vol. 11240, 112405F - (c) 2020 SPIE · CCC code: 1605-7422/20/\$21 · doi: 10.1117/12.2546018 
optical properties of native and coagulated tissue. ${ }^{11}$ The most important factor making PA imaging attractive for RFA is the possibility to integrate this modality with US imaging. ${ }^{12}$ Since both US and PA can use the same transducer, combining these two modalities can provide both structural and functional information. In the case of RFA, clinicians will have both conventional US imaging and additional information from PA imaging about the target structures.

We have previously reported PA imaging using a commercial US system with a light source attached to the transducer. ${ }^{5,13,14}$ We have shown that using this method, we can guide RFA device accurately to a target tissue, visualize blood vessels and differentiate ablated and native tissue. ${ }^{5}$ However, the main limitation of this approach is the penetration depth. Especially for highly absorbing tissue such as liver, illumination from the surface of the tissue is not adequate. ${ }^{13}$ Hence in this work, we propose the use of interstitial illumination based PA imaging for RFA. We use two interstitial illumination methods: (i) an annular fiber probe and (ii) a diffuser fiber. The annular fiber is developed for RFA device guidance. The diffuser fiber is used to image the ablated tissue. We present our preliminary results on ablation monitoring using the diffuser fiber-based PA imaging. We also report an imaging protocol for RFA procedures using the proposed illumination methods.

\section{MATERIALS AND METHODS}

The experimental setup consists of an RFA system, a PA and US imaging system, and a glass tank. Figure. 1 shows the experimental setup. For the PA imaging, the interstitial illumination was used with US detection using a linear array placed outside the sample as shown in Fig. 1a.

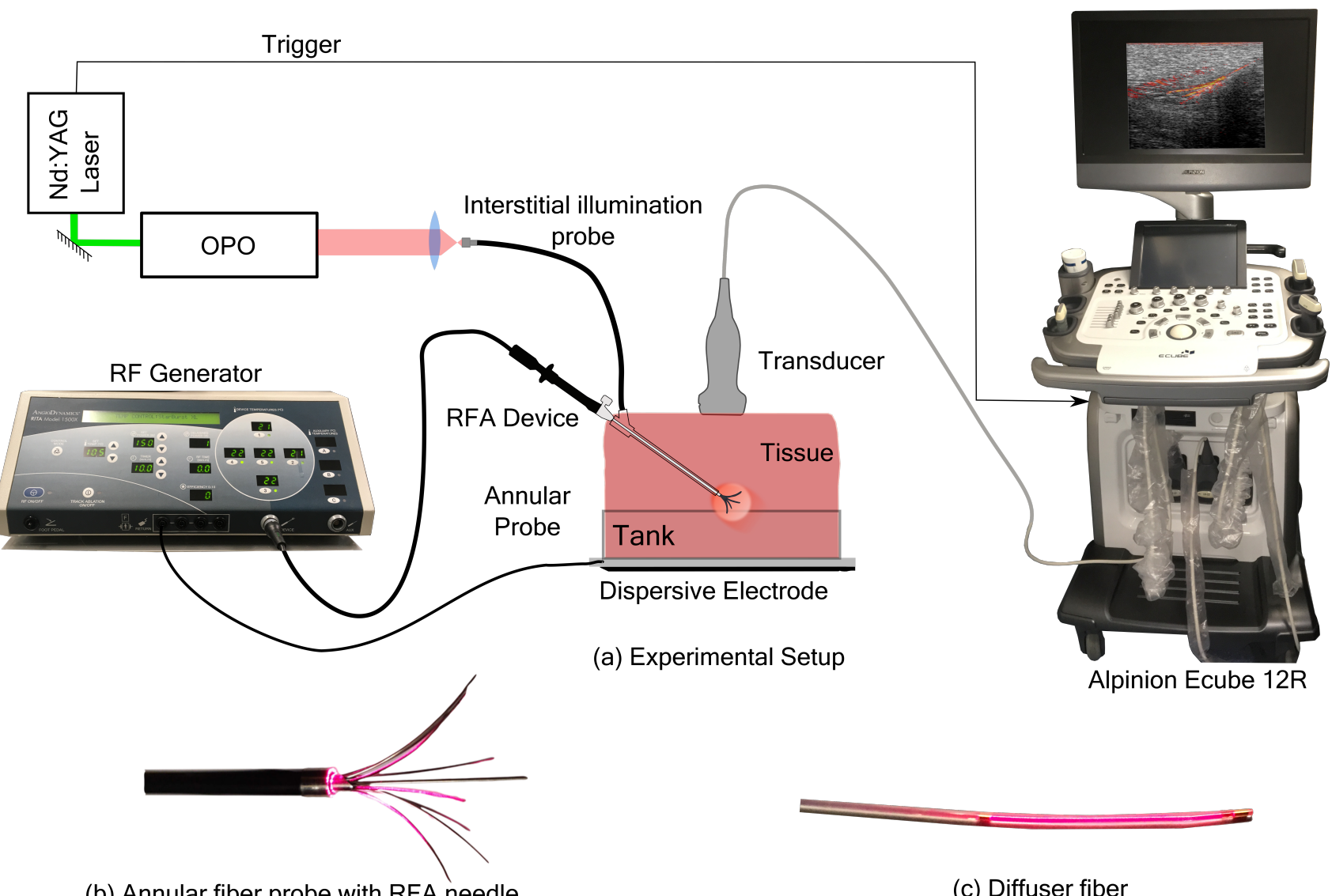

Figure 1. (a) Experimental setup having the RFA system and the imaging system. (b) Annular illumination probe. (c) Diffuser fiber.

The imaging system consists of an Nd:YAG Q-switched laser (Spectra-Physics, USA) and an OPO (versaScan L-532,GWU, Germany) for pulsed PA illumination and a commercially available programmable US system 
Alpinion Ecube 12R (Alpinion Medical Systems, South Korea). The laser with the OPO can be tuned to wavelengths ranging from $680 \mathrm{~nm}$ to $960 \mathrm{~nm}$. The light from the OPO is passed through a series of ND filters to reduce the pulse energy. For both the interstitial illumination methods, we have limited the pulse energy to a maximum of $2 \mathrm{~mJ}$ per pulse at $720 \mathrm{~nm}$. The light is then coupled into the fiber using a lens arrangement.

The annular fiber consists of 72 multimode optical fibers with an NA of 0.22 and a core diameter of $100 \mu \mathrm{m}$. The fibers are bundled at the proximal end for laser coupling, and arranged in an annular fashion at the distal end. The fibers are arranged around stainless-steel ferrule of inner diameter $2.3 \mathrm{~mm}$ and an outer diameter of $3.4 \mathrm{~mm}$. A 14 Gauge RFA needle can be inserted through the lumen of the stainless-steel ferrule as shown in Fig. 1b. The interstitial illumination fiber using the cylindrical diffuser has a $400 \mu \mathrm{m}$ core NA of 0.37 multimode fiber. The fiber is connected to the cylindrical diffuser which has a diameter of $980 \mu \mathrm{m}$ with a length of $30 \mathrm{~mm}$ (Fig. 1c).

PA and US imaging was performed with the Alpinion system. A linear transducer array with a center frequency of $8.5 \mathrm{MHz}$ and a $-6 \mathrm{~dB}$ bandwidth of $3-12 \mathrm{MHz}$ was used for the experiments. A custom sequence was used for combined PA and US imaging. The laser triggered the US system. The imaging sequence consists of a PA signal acquisition in the receive mode followed by a focused B-mode US imaging. A real-time reconstruction algorithm implemented in the system was used for both PA and US imaging.

A clinical RFA system from Angiodynamics (USA) was used in this study. The system consists of an RF generator (1500X, $460 \mathrm{kHz}$ ), a unipolar RFA device (StarBurst XL) and a grounding pad. A glass tank with a metal base was used to simultaneously perform ablation and imaging as shown in Fig. 1a. The grounding pad was attached to the metal base of the tank.

Chicken breast tissue was used for the RFA device guidance experiment. The RFA needle together with the annular fiber probe was inserted into the tissue. Multiple electrodes from the RFA device were then deployed into the tissue. PA and US images of the target tissue were acquired. Illumination of $720 \mathrm{~nm}$ wavelength was considered for the device guidance experiment. Bovine liver tissue was obtained from a local butcher for the ablation experiment. The tissue placed in the glass tank for ablation. The needle RFA device was inserted into the sample and deployed to a radius of $15 \mathrm{~mm}$. The tissue was ablated with a temperature of $105^{0} \mathrm{C}$ for 5 mins. The temperature and duration were selected based on the manufacturer's standard procedure for the specific ablation size. The diffuser fiber was introduced into the ablated region with the help of the biopsy needle cutting through the sample. The diffuser was placed inside the sample and the cutting needle was withdrawn. PA and US images of the ablated region were acquired. A wavelength of $760 \mathrm{~nm}$ was used for PA imaging of the ablated region to target the absorption peak of hemoglobin.

\section{RESULTS AND DISCUSSION}

Figure. 2 shows the RFA device guidance in chicken tissue. The schematic in Fig. 2a shows the location of the annular probe and the RFA device. In the US image in Fig. 2b the RFA needle is partially visible. Only the center electrode of the RFA device is visible along with the top portion of the annular fiber probe. Other electrodes are hard to see under US imaging. 2c shows the PA image of the RFA device. Four electrodes are visible in the PA image. The contrast to noise ratio (CNR) of the PA imaging is also higher than that of the US. A CNR of 9.1 was calculated from the PA image for the electrode at the center while that of the US image it was 1.4. This shows that PA imaging can provide better visualization of the RFA device. This was also verified at different angles. A limitation of the PA image is the artifacts. There are reconstruction artifacts above the needle which are smearing from the back projection. This artifact can be removed with an advanced reconstruction algorithm. ${ }^{15}$ Another issue is the high fluence near the light exiting the fiber, which limits the visibility of low-intensity structures. In the second experiment, an ablated bovine liver was imaged. Figure. 3 shows the results of this experiment. Fig. 3a shows the photograph of the ablated bovine liver sliced through the center of the lesion. The schematic shows the imaging plane with an approximate location of the ablation region with the diffuser fiber placed at the center of the lesion. PA image in Fig. 3c shows a high signal from the native region of the tissue and a slightly lower intensity was observed from the ablated region. This agrees with the change in the optical properties of the tissue with thermal treatment. It was reported that with RFA, optical absorption decreases and scattering increases with thermal treatment compared to the native tissue. This 
Schematic

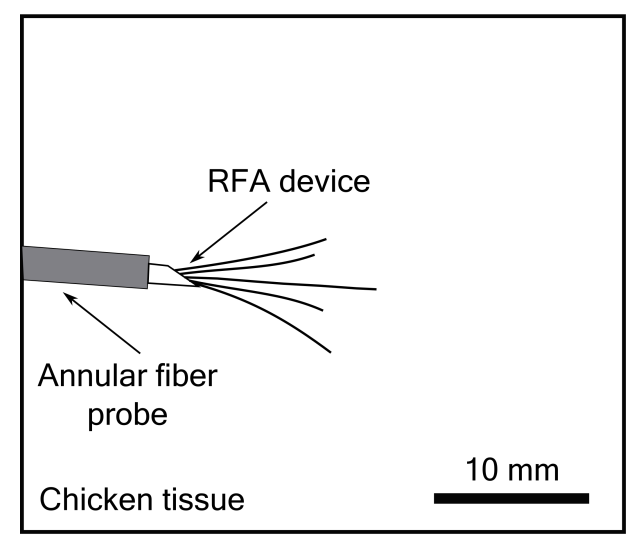

(a)

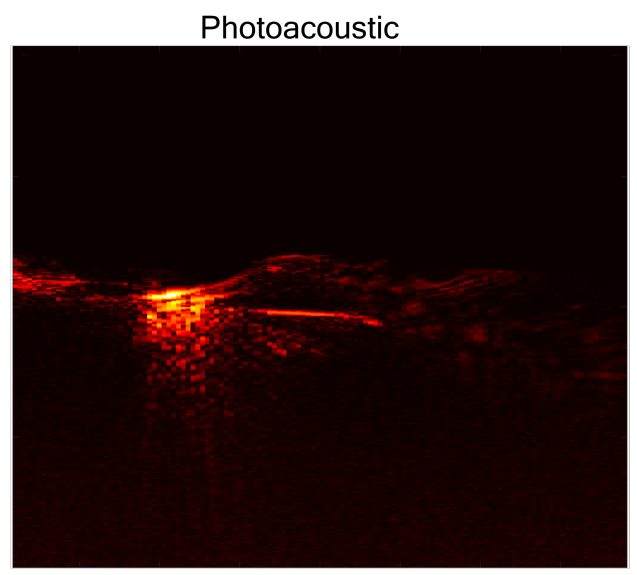

(c)

Photoacoustic

Min

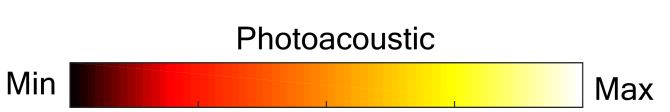

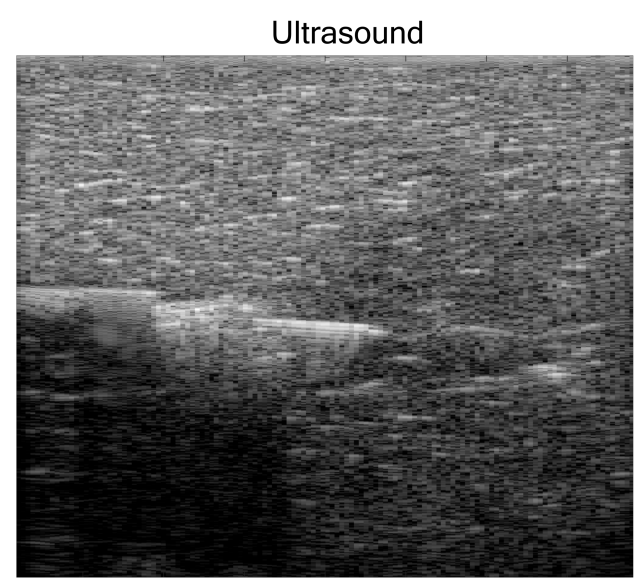

(b)

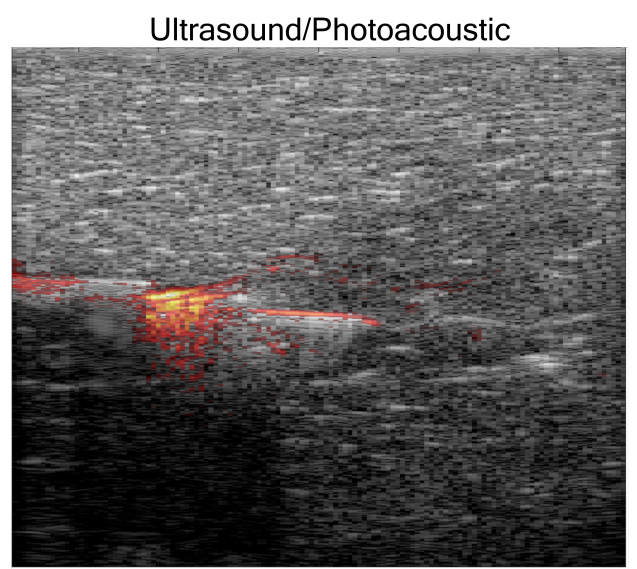

(d)

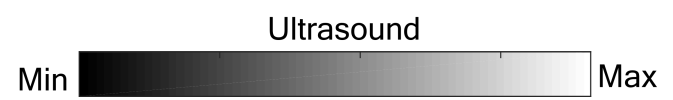

Figure 2. RFA device guidance. (a) A schematic of the imaging plane showing an annular fiber probe and the RFA device in chicken tissue. (b) Ultrasound image of the device and (c) corresponding photoacoustic image.

preliminary result on ablation imaging is promising and we are exploring this approach for an ablated lesion located deep inside the liver.

The two interstitial illumination methods proposed in this work can be used in combination to guide RFA procedures. First, the annular probe with the RFA needle can be inserted into the target tissue under PA and US image guidance. The multiple electrodes of the RFA device can be then inserted into the target volume and this can be verified using PA imaging. After the treatment, the RFA device can be removed and the diffuser fiber can be inserted into the volume to access the thermal lesion. This can be repeated for different locations inside the lesion to verify the complete ablation of the target tissue.

\section{CONCLUSION}

Based on our preliminary results we have shown that photoacoustic imaging can be potentially used for RFA procedures. Primarily, the proposed approach can be used for accurate RFA device guidance and assessing the extent of the ablation lesion. The technology is still at an early stage to claim any clinical relevance. However, based on experiments performed in a laboratory setting, photoacoustic imaging has shown the potential to provide added information to ultrasound imaging. 


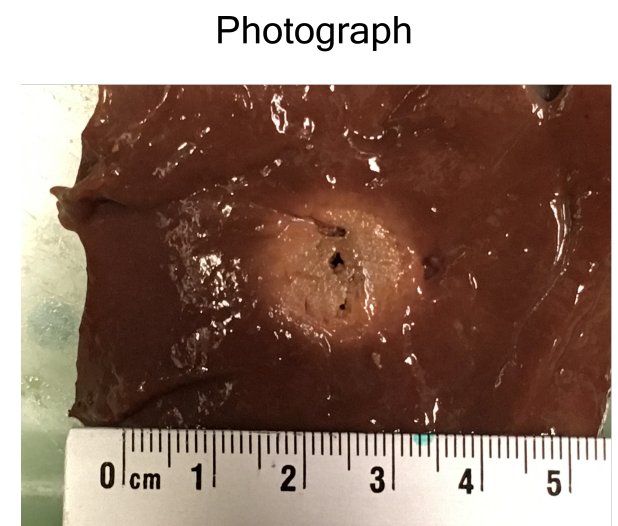

(a)

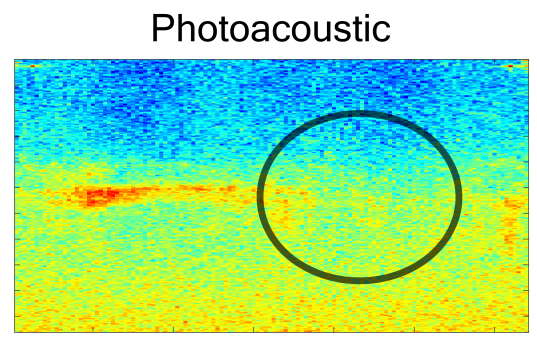

(c)

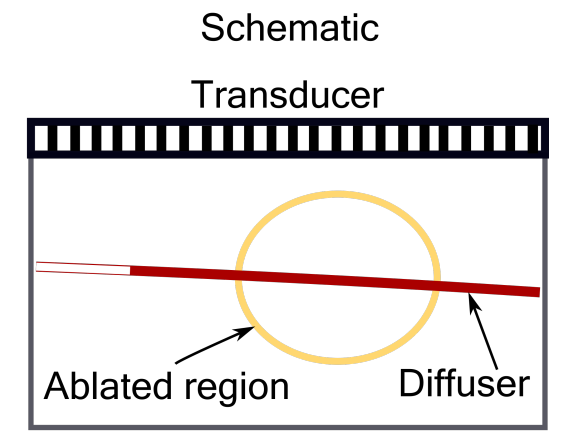

(b)

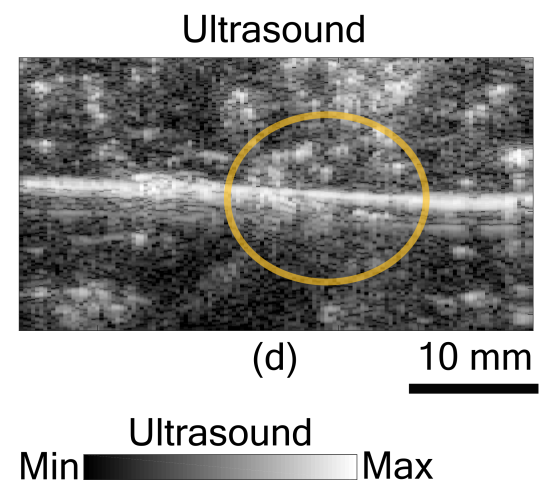

Figure 3. Ablation imaging. (a) Photograph of the bovine tissue sliced through the ablated region. (b) A schematic of the imaging plane with the transducer on the top of the sample, diffuser fiber placed through the center of the ablated region with the ablated region marked with a yellow circle. (c) Photoacoustic image of the tissue and (d) the corresponding ultrasound image.

\section{ACKNOWLEDGMENTS}

This work is funded by a joint grant from the Netherlands Organisation for Scientific Research (NWO)/the Netherlands Organisation for Health Research and Development (ZonMw) and the Department of Biotechnology (Government of India) under the program Medical Devices for Affordable Health (MDAH) as Project Imaging Needles (Grant Number 116310008).

\section{REFERENCES}

[1] Si, M.-B., Yan, P.-J., Hao, X.-Y., Du, Z.-Y., Tian, H.-W., Yang, J., Han, C.-W., Yang, K.-H., and Guo, T.-K., "Efficacy and safety of radiofrequency ablation versus minimally invasive liver surgery for small hepatocellular carcinoma: a systematic review and meta-analysis," Surgical endoscopy , 1-11 (2019).

[2] Rhim, H., Goldberg, S. N., Dodd, G. D., Solbiati, L., Lim, H. K., Tonolini, M., and Cho, O. K., "Essential techniques for successful radio-frequency thermal ablation of malignant hepatic tumors," Radiographics 21(suppl_1), S17-S35 (2001).

[3] Lencioni, R., Cioni, D., Crocetti, L., Franchini, C., Pina, C. D., Lera, J., and Bartolozzi, C., "Earlystage hepatocellular carcinoma in patients with cirrhosis: long-term results of percutaneous image-guided radiofrequency ablation," Radiology 234(3), 961-967 (2005).

[4] Lee, E. W., Loh, C. T., and Kee, S. T., "Imaging guided percutaneous irreversible electroporation: ultrasound and immunohistological correlation," Technology in cancer research 85 treatment 6(4), 287-293 (2007).

[5] Francis, K. J. and Manohar, S., "Photoacoustic imaging in percutaneous radiofrequency ablation: device guidance and ablation visualization," Physics in Medicine 63 Biology 64(18), 184001 (2019). 
[6] Wang, D., Wu, Y., and Xia, J., "Review on photoacoustic imaging of the brain using nanoprobes," Neurophotonics 3(1), 010901 (2016).

[7] Manohar, S. and Dantuma, M., "Current and future trends in photoacoustic breast imaging," Photoacoustics (2019).

[8] Francis, K. J., Chinni, B., Channappayya, S. S., Pachamuthu, R., Dogra, V. S., and Rao, N., "Characterization of lens based photoacoustic imaging system," Photoacoustics 8, 37-47 (2017).

[9] Su, J. L., Karpiouk, A. B., Wang, B., and Emelianov, S. Y., "Photoacoustic imaging of clinical metal needles in tissue," Journal of biomedical optics 15(2), 021309 (2010).

[10] Upputuri, P. K., Sivasubramanian, K., Mark, C. S. K., and Pramanik, M., "Recent developments in vascular imaging techniques in tissue engineering and regenerative medicine," BioMed research international 2015 (2015).

[11] Lanka, P., Francis, K., Kruit, H., Sekar, S. K. V., Farina, A., Cubeddu, R., Manohar, S., and Pifferi, A., "Monitoring radiofrequency ablation of biological tissue using broadband time-resolved diffuse optical spectroscopy," in [European Conference on Biomedical Optics], 11074_94, Optical Society of America (2019).

[12] Singh, M. K. A., Steenbergen, W., and Manohar, S., "Handheld probe-based dual mode ultrasound/photoacoustics for biomedical imaging," in [Frontiers in Biophotonics for Translational Medicine], 209-247, Springer (2016).

[13] Francis, K. J., Rascevska, E., and Manohar, S., "Photoacoustic imaging assisted radiofrequency ablation: Illumination strategies and prospects," in [TENCON 2019-2019 IEEE Region 10 Conference (TENCON)], 118-122, IEEE (2019).

[14] Rascevska, E., Francis, K. J., and Manohar, S., "Annular illumination photoacoustic probe for needle guidance in medical interventions," in [European Conference on Biomedical Optics], 11077_20, Optical Society of America (2019).

[15] Mozaffarzadeh, M., Mahloojifar, A., Orooji, M., Kratkiewicz, K., Adabi, S., and Nasiriavanaki, M., "Lineararray photoacoustic imaging using minimum variance-based delay multiply and sum adaptive beamforming algorithm," Journal of biomedical optics 23(2), 026002 (2018). 